\title{
REGISTER OF EVENTS
}

\section{- SOCIETY FOR BACK PAIN RESEARCH} 5 March, 1993, England

Secr.: Jacqui Robertshaw, 30 Queen Street, Huddersfield HD1 2 SP England

Tel.: 0484424329

- XXXVIIIC JOURNEES ANNUELLE DU CENTRE VIGGO PETERSEN - L'ACTUALITE RHUMATOLOGIQUE 1993

18-19 mars 1993, Paris, Palaiș des Congrès, France.

Secr.: Mme C. Moroy, Centre Viggo Petersen, Clinique de Rhumatologie, Hôpitel Lariboisière, 6 rue Guy Patin, F-75010 Paris, France.

Tél.: 49.95.62.90 - Fax: 49.95.86.31

- IIIrd INTERNATIONAL SYMPOSIUM ON ANKYLOSING SPONDYLITIS

HLA-B27 - Twenty Years On

March 30-31, 1993, Middlesex Hospital, London

Secr.: Dr. Alan Ebringer, Dept Rheumatology, Middlesex Hospital, LONDON W.1, UK.

- THE SECOND INTERNATIONAL SYMPOSIUM ON THE IMMUNOTHERAPY OF THE RHEUMATIC DISEASES

Brighton, England. 1-4 April 1993.

Secr.: Professor G.S. Panayi, The Rheumatology Unit, Division of Medicine, 4th Floor Hunts House, UMDS, Guy's Hospital, London, SE1 9RT, UK

- THE XXIIIrd EUROPEAN SYMPOSIUM ON CALCIFIED TISSUES

Heidelberg, Germany, April 25-29, 1993

Prof. Dr. Ziegler, ESCT, Medizinische Universitätsklinik

Bergheimer Str. 58, W-6900 Heidelberg, Germany

- 5th INWIN 93. INTERSCIENCE WORLD CONFERENCE ON INFLAMMATION, ANTIRHEUMATICS, ANALGESICS, IMMUNOMODULATORS

Palexpo Geneva, Switzerland, 25-28 April 1993.

Secr.: 5th INWIN, P.O. Box 112, 1218 Grand Saconnex (GE), Switzerland

- SIXTH INTERNATIONAL CONFERENCE ON BEHCET'S DISEASE

Paris, France, June 30-July 1, 1993

Secr.: Bertrand Wechsler MD, Pitié-Salpêtrière Hospital, 47/83 Bd de l'Hôpital, 75013 Paris Cedex 13 France.

- XVIIIth ILAR CONGRESS OF RHEUMATOLOGY July 4th-10th, 1993, Barcelona (Spain)

Secr.: Viajes Iberia, Congresos, Diagonal, 523, $1^{\circ}, 08029$ Barcelona.

\footnotetext{
- 2nd EULAR WORKSHOP ON CARTILAGE AND BONE RESEARCH
}

University of Gothenburg

3-5 September 1993, Gothenburg, Sweden

Secr.: Mrs Barbro Tylén, Dept. of Rheumatology, Sahlgren

Univ Hospital, S-413 45 Göteborg, Sweden.

Fax : 46.31.417938

- XI PANAMERICAN CONGRESS OF RHEUMATOLOGY. XX CONGRESSO BRASILEIRO DE REUMATOLOGIA September 18-23, 1994, Recife - Pernambuco, Brasil Secr.: LK Assessoria E Promoçóes LTDA, Rua Costa Pereira 9 - Tijuca, 20511 - Rio de Janeiro - RJ - Brazil

\section{- INTERNATIONAL CONGRESS ON OBESITY MAN-} AGEMENT

Antwerp, Belgium, 19-22 September 1993.

Secr.: Miep Bekkers-van den Hove, Obesitas vzw, Bunderbeeklaan 19, B-2950 Kapellen - Belgium

- Vth PRAGUE RHEUMATOLOGICAL SYMPOSIUM 19-23 September 1993, Prague, Czechoslovakia Secr.: Na slupi 4, 12850 Prague 2, Czechoslovakia

\section{- SECOND EUROPEAN CONFERENCE ON SYSTEMIC} LUPUS ERYTHEMATOSUS

October 28-30 1993 - Erlangen, University of ErlangenNürnberg

Secr.: Mrs. Kauntz/Mrs Herbach, Department of Medicine III and Institute for Clinical Immunology and Rheumatology, Krankenhausstr. 12, D-8520 Erlangen

Tel.: 09131/85.91.31 - Fax : 09131/85.47.70

\section{- 4th ASEAN CONGRESS OF RHEUMATOLOGY} 31st Oct 1993 - 4th Nov 1993, Singapore

Secr.: 4th Asean Congress of Rheumatology, 336 Smith Street \#06-302, New Bridge Centre, Singapore 0105, Republic of Singapore.

Tel.: 65.2279811 - Fax : 65.2270257

\section{- NINTH WORKSHOP ON VITAMIN D} May 28 - June 2, 1994, Orlando, Florida, USA

Secr.: Vitamin D Workshop Secretary, Biochemistry Department

University of California, Riverside, Ca. 92521-0129, USA Fax : 714.787-4784

- VIII EULAR SYMPOSIUM VASCULITIS

June, 8-11, 1994, Istanbul, Turkey

Secr.: A\&B Public Relations, Valikonaği Caddesi, 46/6 Nişantaş 80200 Istanbul - Turkey.

- THE 2ND APLAR SYMPOSIUM ON THE THERAPY OF RHEUMATIC DISEASES

December 1994, Kuala Lumpur, Malaysia

Secr.: 2nd APLAR Symposium on The Therapy of Rheumatic Diseases, c/o Ciba-Geigy Pharma Division, P.O. Box 1005, Jalan Semangat, 46860 Petaling Jaya, Malaysia. 